\title{
Criminologie
}

\section{Réflexions méthodologiques pour une recherche sur l'opinion publique et l'action sociale : essai de synthèse}

\section{Christiane Louis-Guérin}

Volume 12, numéro 1, 1979

Justice et public

URI : https://id.erudit.org/iderudit/017097ar

DOI : https://doi.org/10.7202/017097ar

Aller au sommaire du numéro

Éditeur(s)

Les Presses de l'Université de Montréal

ISSN

0316-0041 (imprimé)

1492-1367 (numérique)

Découvrir la revue

Citer cet article

Louis-Guérin, C. (1979). Réflexions méthodologiques pour une recherche sur l'opinion publique et l'action sociale : essai de synthèse. Criminologie, 12(1),

24-42. https://doi.org/10.7202/017097ar d'utilisation que vous pouvez consulter en ligne. 
RÉFLEXIONS MÉTHODOLOGIQUES POUR UNE RECHERCHE SUR L'OPINION PUBLIQUE ET L'ACTION SOCIALE : ESSAI DE SYNTHESE

Christiane Louis-Guérin

L'opinion publique intéresse tous ceux qui de près ou de loin s'occupent de politique au sens large du terme. Machiavel conseillait déjà, si l'on veut être un chef avisé, de ne jamais ignorer l'opinion publique dans certaines des décisions. Il faut attendre toutefois le $18^{\mathrm{e}}$ siècle pour que l'opinion publique devienne un élément important dans la politique.

Bien que des études systématiques sur l'opinion publique soient réalisées depuis la Première Guerre mondiale, ce n'est qu'après la Seconde Guerre que les sondages d'opinions vont connaître un véritable essor. Cet essor est dû notamment aux travaux de Lazarsfeld et de Hyman à l'Université de Columbia et de Stouffer à Harvard. Au début des années cinquante, les méthodes d'enquêtes vont dominer la sociologie américaine et restent encore actuellement une des approches les plus utilisées dans la recherche. Parallèlement à ces développements universitaires, de nombreux centres de sondages, para-universitaires, privés ou publics vont être crés. Présentement les sondages d'opinions font partie de la réalité politique et quotidienne sinon l'influencent; chaque jour et dans tous les domaines de la vie publique ou privée on sonde et on mesure les opinions et les attitudes des gens.

Il est certain que l'apparente facilité et rigueur des techniques de sondages et leur capacité de tester rapidement et économiquement l'opinion publique sur presque n'importe quel problème, ont contribué à cette prolifération que l'on observe. Toutefois de plus en plus de chercheurs remettent en question non seulement l'utilité et l'usage que l'on fait des résultats ainsi obtenus, mais également critiquent les limites mêmes de cette approche pour comprendre le comportement social.

Dans cet article nous voudrions indiquer, à partir des limites mêmes des sondages d'opinions et des enquêtes d'attitudes, comment certaines approches, notamment : l'enquête contextuelle, l'étude des représentations sociales et l'ethnométhodologie, ont cherché à développer des outils conceptuels et méthodologiques plus adéquats. Nous tenterons en particulier de montrer que, 
malgré leurs différences apparentes, ces trois approches peuvent être utilement intégrées. Une telle synthèse éventuelle nous semble pouvoir fournir un cadre heuristique pour comprendre l'organisation et les actions sociales.

\section{Opinion publique et mesures des opinions}

\subsection{Opinion publique}

Bien que les sondages d'opinions visent principalement à déterminer les différentes opinions publiques sur un problème donné, il ne semble guère y avoir de consensus sur la notion même d'opinion publique. En effet on trouve à l'une des extrémités du continuum, des définitions proches de la notion durkheimienne de pensée collective, et à l'autre extrémité, par contraste, des définitions strictement opérationnelles telle que \& l'opinion publique est un complexe de préférences exprimées par un nombre significatif de personnes sur un problème d'importance générale ${ }^{1}$ 》.

Différents facteurs constitutifs sont nécessaires pour qu'il existe une opinion publique. Tout d'abord, l'existence d'une situation problématique sur laquelle peuvent se prononcer les gens et par laquelle ils peuvent avoir des avis divergents. L'élément de controverse doit donc être nécessairement impliqué. Deuxièmement, le problème doit concerner un groupe reconnaissable de personnes. C'est ce qu'on appellera le public de l'opinion publique. Ainsi, on parlera du public des consommateurs, des votants, etc. Troisièmement, l'opinion publique réfère à la totalité des opinions qui existent dans le public sur un problème donné. Il faut tenir compte non seulement de la distribution des opinions en termes de direction et d'intensité, mais également de toutes les autres opinions individuelles, mesurées ou non, qui sont directement reliées à la manière dont est jugé et/ou évalué le problème. Autrement dit l'opinion publique n'est pas seulement un ensemble de prises de position sur une question problématique, mais inclut implicitement ou explicitement les multiples raisons individuelles qui sous-tendent ces différentes prises de position. Quatrièmement, pour qu'il existe une opinion publique, il faut que le public concerné soit assez nombreux et d'autre part que le problème soit d'intérêt public. En effet, de petits groupes de gens

1. Hennesy, 1975 , p. 5. 
concernés essentiellement par des questions personnelles, peu importantes ou privées, ne peuvent constituer une opinion publique. L'opinion publique doit pouvoir exercer une influence sur le comportement individuel, le comportement des groupes et les politiques gouvernementales.

\subsection{Sondages d'opinions}

Nous ne nous étendrons guère sur la méthode des sondages courants qui se limitent le plus souvent à recueillir les réactions d'un public sur une question particulière d'actualité ou d'intérêt, à travers une ou quelques questions dont on vérifie rarement la pertinence et la compréhension pour les différents répondants. Les analyses ensuite se bornent à mettre en relation la ou les réponses ainsi obtenues avec certaines caractéristiques de la population. Le caractère illusoire d'une opinion publique ainsi mesurée a été très largement démontré. On peut indiquer certaines des critiques majeures, en particulier on reproche aux sondages d'opinions de formuler des questions très générales et abstraites alors que les gens réagissent et agissent en fonction de situations particulières. Ces questions en outre reflètent plus souvent la problématique du chercheur ou des demandeurs plutôt que celle des répondants, les questions posées n'étant pas forcément celles que se posent les gens et/ou de la façon dont ils se les posent. On tient rarement compte que les phénomènes ont une visibilité et une pertinence souvent distinctes d'un groupe à l'autre et que les différentes classes sociales maîtrisent plus ou moins bien certaines questions problématiques et sont plus ou moins aptes à générer une contre-problématique ${ }^{2}$. Dans la mesure où l'on considère que les gens ont réponse à tout, souvent le même poids est accordé aux opinions quelles que soient la position et la situation sociales des répondants, sans mentionner le fait que l'on espère que les gens partagent la même définition de la situation et donc que leurs réponses ont le même sens. De nombreuses recherches ont montré la disparité des codes linguistiques ${ }^{3}$ et des modes de pensée " selon les classes sociales. Dans une société, comme la société multiculturelle canadienne, cet aspect apparaît d'autant plus pertinent. Vouloir donc uniformiser selon ce qu'on appelle

2. Bourdieu, 1973.

3. Bernstein, 1964.

4. Schatzman et Strauss, 1955. 
4 le plus petit dénominateur commun $\$$, la formulation de questions qui quelquefois recouvrent une réalité complexe, conduit à déformer les opinions réelles des gens. Faut-il rappeler, par exemple, que deux personnes également opposées à la peine de mort peuvent en fait avoir des conceptions très différentes sur son sens, les causes et conséquences ainsi que les actions à entreprendre. Au niveau de l'analyse le fait de se limiter à des relations entre les variables prises deux à deux masquent certains phénomènes. En effet les corrélations ainsi observées peuvent être expliquées par d'autres variables intermédiaires que le simple tri croisé ne permet certes pas de tester. On peut aussi reprocher aux sondages d'opinions qui utilisent des variables sociologiques tels que les jeunes, les Québécois, les femmes et les hommes, les Noirs, etc., de renforcer les stéréotypes plutôt que de refléter une réalité sociale. En outre cette présentation accentue souvent la polarisation apparente des opinions.

Parler d'opinion publique dans cette perspective semble alors naïf ou relevant de la manipulation. Il serait intéressant, à ce propos, d'étudier le rôle de tels sondages dans la création, le renforcement ou la transformation des opinions. En effet dans la mesure où ils proposent une certaine interprétation de la réalité sociale en réfractant * apparemment » les opinions publiques, ils peuvent devenir à leur tour un élément de signification sur lequel se basent les gens pour interpréter leurs actions et les actions des autres.

Si l'on considère que l'opinion publique, comme nous l'avons indiqué, se caractérise en particulier par sa faculté à s'organiser et à se cristalliser en une force capable d'orienter le changement, on peut difficilement, sur la base de certaines réponses ponctuelles à une ou à quelques questions, dont la fiabilité en outre n'est guère établie, parler de mobilisation des opinions ou d'un consensus social. Ce qu'on appelle ici opinion publique n'est le plus souvent qu'un agrégat d'individus qui * apparemment » ont les mêmes opinions. Egalement dans le cas où il semble exister * apparemment $\gg$ un consensus on peut se demander ce qu'il signifie. Comme le souligne Bourdieu ${ }^{5}$, les catégories sociologiques choisies le sont souvent sans fondement théorique, tel le statut socioEconomique. Les relations entre ce statut et la classe sociale ren-

5. Bourdieu, 1965. 
voient aux problèmes notamment de conscience de classe et de fausse conscience. Dans nos sociétés, dites de communication de masse, notamment le continent nord américain, l'individu saturé par les media est dans une certaine mesure, aliéné, ce que Marcuse a appelé l' homme unidimensionnel \$. Les études, sur la communication et l'influence des media, ont montré que la grande majorité des gens se sentent, en fait, peu concernés par les affaires sociales et n'ont que peu d'opinions sur les grands problèmes politiques et sociaux. Ils connaissent mal, sinon pas du tout, les divers programmes politiques, même lorsqu'il s'agit d'un vote présidentiel. Parler de consensus, dans ce contexte, réfère davantage à ce que Machiavel appelle «la tolérance passive des masses » plutôt qu'un support positif du public.

\subsection{Enquêtes d'attitudes}

Les enquêtes d'attitudes vont chercher à dépasser le niveau manifeste des opinions pour atteindre un niveau plus profond de structuration, à savoir les attitudes. On distingue ainsi l'opinion, qui est une donnée observable, de l'attitude qui est un construit hypothétique, inféré à partir des relations entre les opinions. D'une manière générale on a pu montrer que l'opinion est peu stable, à la différence de l'attitude.

Opérationnellement l'opinion constitue une réponse simple et manifeste à une question, alors que l'attitude est inférée à partir d'un ensemble de réponses organisées selon une logique interne propre. Les données du premier degré, que l'on obtient dans un questionnaire d'attitudes, sont donc des opinions. Pour atteindre les attitudes, données du second degré, il s'agit de chercher les principes organisateurs liant ensemble respectivement un certain nombre d'opinions référant à un même domaine d'attitudes. Comme Moscovici (1961) le souligne il ne s'agit pas de croire qu'on «donne telle réponse parce qu'on a telle attitude ». On n'explique rien dans ce cas-là : l'attitude est explicative seulement si elle-même est expliquée, c'est-à-dire si on peut définir les déterminations qui la fondent et dont elle est une synthèse ou un carrefour ${ }^{6} \gg$. Il apparaît donc, dans cette perspective, essentiel de s'assurer non seulement de la pertinence des questions, de leur exhaustivité par rapport au champ étudié, mais éga-

6. Moscovici, 1961, p. 267. 
lement, il s'agit d'élaborer un schème conceptuel, permettant de reconstituer de façon non ambiguë, l'ensemble des attitudes relatives au champ étudié. Enfin il est nécessaire d'identifier les différents déterminants qui fondent les attitudes.

Ces trois opérations sont fondamentales dans une enquête d'attitudes. En particulier il est important, si l'on ne veut pas se limiter à des opinions, qui en elles-mêmes n'ont que peu de sens, de développer une problématique rigoureuse.

\subsection{Démarche itérative qualitative-quantitative}

Une recherche d'exploration intensive des phénomènes s'impose dans la majorité des cas. Elle a pour but non seulement de permettre la construction d'un instrument et des mesures fiables, d'affiner les hypothèses et d'en développer de nouvelles, de recentrer la problématique, mais elle peut également être utilisée pour expliciter certaines relations statistiques observées dans l'enquête et difficiles à interpréter. Elle éclaire alors les résultats quantitatifs en se servant des données de l'expérience vécue. En explorant la signification subjective des comportements, c'est-àdire les intentions réelles des acteurs, on peut espérer mettre en évidence des éléments nouveaux guère évidents au départ. Cette démarche en "feed back» offrirait l'opportunité de passer d'une forme de données à une autre et vice versa. Dans cette perspective, au lieu de reléguer l'approche qualitative à une fonction mineure d'exploration, elle devient une recherche en elle-même visant une modélisation des comportements. Le modèle concret ainsi établi peut ensuite être testé extensivement par l'enquête quantitative et en même temps prolonge en le complétant le modèle abstrait établi par l'enquête. On n'a guère exploité cette opportunité conciliant réellement deux approches que l'on a voulu trop souvent opposer. Une telle démarche pourrait peutêtre pallier certaines des limites inhérentes à ces deux approches, en particulier celles de l'enquête d'attitudes dont nous présentons maintenant les faiblesses majeures.

\subsection{Limites des enquêtes d'attitudes}

En effet les enquêtes d'attitudes n'échappent guère aux critiques. On leur reproche notamment leur centration exclusive sur les comportements individuels, négligeant le milieu actuel dans lequel vivent les répondants. Également la logique même des 
méthodes quantitatives est souvent mise en question, en particulier celle des échelles et de l'analyse des variables. Cicourel (1964), par exemple, considère les questions fermées et précodées comme un véritable * anathème ». Elles imposent un rôle passif d'* acceptation » au répondant qui doit réagir à des catégories préconçues par le chercheur. En outre, on peut se demander à quelle réalité de conscience, correspondent des catégories imposées comme plus ou moins * favorables », etc., sans mentionner les mesures utilisées de rang, d'intensité, d'intervalle ou autre. Les traitements statistiques ne sont guère non plus des procédures neutres, libres de préjugés. Les statistiques, en effet, en contenant des principes de distribution des phénomènes, imposent aussi un modèle implicite de l'homme et de l'action.

Mais un des aspects, peut-être le plus problématique des techniques conventionnelles, porte sur l'ordre et le modèle qu'elles imposent aux phénomènes étudiés, créant ainsi des significations extrinsèques. Ainsi sans mentionner l'ordonnance des questions et leur formulation qui déterminent en partie les réponses au niveau même de l'explication des biais peuvent exister. En effet lorsque l'on veut expliquer le comportement théorique des acteurs, on procède selon une reconstruction logique des motifs, qu'ils soient psychologiques et/ou sociologiques. L'enchaînement des actions est alors expliqué en fonction des hypothèses et des questions posées dont on suppose la pertinence. Nous n'entrerons guère dans les querelles soulevées par l'analyse des variables? identifiées à partir d'indices objectifs pour déterminer leur existence réelle. Notons toutefois que l'individu est considéré ici comme un véhicule passif à travers lequel les variables opèrent, celles-ci renvoyant soit à des qualités stables intériorisées, soit à des mécanismes externes de contrôle social. Cette conception déterministe du comportement humain, mettant l'accent sur la cohérence et l'adaptation passive, reste statique. En effet, le changement «ne peut plus surgir que d'une conversion morale et/ou d'une évolution impersonnelle dont les racines - pour dire le moins - restent toujours mystérieuses ${ }^{8}$.

En outre le chercheur passe d'une conception générale : les normes, les valeurs, etc., aux expressions individuelles saisies à travers les questions d'attitudes, pour de nouveau repasser à

7. Blumer, 1956.

8. Crozier et Friedberg, 1977, p. 175. 
des conceptions générales telles que « opinion publique 》, 《consensus », etc., qui, en fait, expriment des opinions, des sentiments individuels d'un agrégat de personnes, dont la réalité en tant que groupe capable d'action collective reste très problématique.

Le récent paradigme interactif notamment, récuse ce raisonnement déterministe et statistique du sens des actions. Dans cette orientation le sens, en revanche, est considéré comme un produit émergent de l'interaction qui dépend $e t$ des caractéristiques de la situation et des caractéristiques des interactions. On ne peut donc comprendre le comportement en dehors du contexte particulier dans lequel il s'actualise. L'étude de Lapierre (1934) est un des exemples célèbres sur les liens faibles entre les attitudes mesurées et le comportement réel qui peut varier d'une situation à une autre.

La notion d'attitude telle que conçue traditionnellement et les méthodes conventionnelles pour mesurer ces attitudes apparaissent ainsi de plus en plus inadéquates pour comprendre les conduites. L'enquête contextuelle, l'étude des représentations sociales et la sociologie phénoménologique, notamment l'ethnométhodologie, ont développé chacune de leur côté, une approche pour dépasser les limites des enquêtes d'attitudes.

2. Trois approches pour l'étude des conduites sociales : vers une tentative de synthèse

Nous voudrions montrer, malgré leurs différences, que ces trois approches peuvent se compléter avec profit et offrir ainsi un cadre heuristique plus riche pour l'étude des conduites sociales. Après avoir présenté leur démarche spécifique, on proposera une tentative d'articulation possible pour intégrer ces différentes approches.

\subsection{Enquête contextuelle}

On ne peut nier que les contraintes et les opportunités du milieu dans lequel on vit ont un certain impact sur nos conduites. Comme le souligne Boudon (1971) :

Sociologiquement un individu n'est pas seulement un complexe de variables individuelles, il appartient aussi à un certain milieu et ce sont souvent les caractéristiques de ce milieu qui expliquent son comportement plutôt que ces 
caractéristiques dites « sociologiques » également individuelles (sexe, âge, statut socio-économique, etc.) ${ }^{9}$

Blumer (1954) indique aussi le caractère « asociologique » de la presque totalité des sondages qui n'étudient l'individu que comme une abstraction détachée de son contexte social. Les mesures classiques qui portent sur un ensemble agrégé d'individus n'apportent que très peu d'informations sur le milieu dans lequel s'insèrent ces individus. Même si l'on tient compte de la classe sociale, de la profession, etc., on ne peut expliquer l'ensemble des attitudes et des conduites sociales qui peuvent dépendre de nombreux autres facteurs liés à la structure sociale. Par exemple, Lipset et al. (1956) étudiant le comportement syndical d'ouvriers typographes ont montré qu'il est souvent conditionné par les caractéristiques de l'entreprise, le climat social, etc. Cette actuelle coupure dans la grande majorité des études qui existent entre le niveau microsociologique et macrosociologique, conduit à une situation paradoxale. En effet, malgré l'accumulation de données empiriques, on connaît encore très mal l'impact réel des structures sociales sur le comportement.

Un des exemples certainement les plus frappants de ces dernières années fut la surprise des sociologues devant les contestations étudiantes. Pourtant on ne peut guère dire que les étudiants soient un groupe négligé par la recherche, bien au contraire ils ont toujours constitué la cible favorite de la sociologie ou psychologie universitaire. Les explications développées à posteriori de cette crise sociale restent également très souvent insuffisantes, soit parce qu'elles se situent d'emblée au niveau macrosociologique ${ }^{10}$ ou bien restent strictement au niveau microsociologique ${ }^{11}$.

Le passage, entre le niveau microsociologique auquel s'adressent les enquêtes traditionnelles et le niveau macrosociologique auquel se situe la crise sociale, est difficile. Mais il est tout aussi difficile de saisir directement les phénomènes macrosociologiques sans étudier en même temps leurs effets sur les conduites individuelles.

L'analyse contextuelle développée par Lazarsfeld (1972) va permettre de concilier ces deux niveaux de signification. Il a

9. Boudon, 1971 , p. 57.

10. Voir Touraine, 1972.

11. Voir Klineberg, Louis-Guérin et Zavalloni, 1978. 
repris la logique des sondages centrés sur les individus pour l'étendre à des unités autres que les individus. Ce double plan d'observation, où l'on peut saisir simultanément les caractéristiques « collectives » (revenus moyens de la communauté, son taux de criminalité, distribution des agences socioculturelles et pénales, etc.) de l'environnement immédiat dans lequel vivent les individus étudiés et en même temps leurs caractéristiques individuelles (classe sociale, âge, opinion, actions, etc.), permet alors d'établir les liens qui existent entre les conditions de vie réelles et les conduites. On remplace ainsi un modèle atomiste n'incluant que des variables définies sur des individus par un modèle contextuel incluant simultanément des variables individuelles et collectives.

Toutefois cette perspective a des limites également, outre les faiblesses inhérentes aux méthodes d'enquête. En effet bien que l'on puisse spécifier l'environnement externe d'une personne en termes « objectifs » indépendamment de la perspective particulière de l'acteur, cet environnement en fait n'est pas perçu et expérimenté comme tel par l'acteur, mais reconstruit, interprété selon des modalités particulières. C'est à partir de cette construction de la réalité que les actions se déterminent. L'influence des facteurs culturels, sociaux, économiques, politiques et physiques sur les conduites n'est donc pas directe, mais dépend en grande partie de médiations qui structurent la situation. Ces médiations entre autres sont les représentations sociales ou construits collectifs des objets sociaux par un groupe ou une classe sociale. Ils contiennent en même temps l'idéologie dominante par rapport à laquelle ils s'élaborent et des éléments de différenciation renvoyant aux relations particulières qu'entretiennent avec le réel ces différents groupes ou classes sociales.

\subsection{Représentations sociales}

L'étude des représentations sociales va chercher à identifier le champ idéologique dans lequel et par rapport auquel se situe l'action individuelle et collective. Dans cette perspective on va mettre l'accent sur l'étude des rapports entre l'individu et l'objet caractérisé comme élément d'un système social. On cherche, en effet, à définir ce que représente l'objet dans le contexte socioculturel propre à chaque répondant, en même temps que ses réactions envers cet objet. 
Pour Moscovici (1961, 1963), le premier qui a développé systématiquement cette notion, la représentation réfère à la construction d'un objet par une communauté dans le but d'agir et de communiquer. Comme il l'indique, elle est « un système de valeurs, de notions et de pratiques ayant une double vocation : tout d'abord, d'instaurer un ordre qui donne aux individus la possibilité de s'orienter dans l'environnement social, matériel et de le dominer. Ensuite d'assurer la communication entre les membres d'une communauté en leur proposant un code pour leurs échanges et un code pour nommer et classer de manière univoque les parties de leur monde, de leur histoire individuelle ou collective ${ }^{12} \gg$.

Les représentations, pour cet auteur, reflètent donc un \&univers d'opinions $\gg$ en tant qu'organisation stable de perceptions et de connaissances relatives à un certain aspect du monde de l'individu ou du groupe. Elles dépendent de la structure sociale et de la situation particulière de l'individu ou du groupe dans cette structure, c'est-à-dire de la proximité au pouvoir et au système de décisions, des pratiques sociales et de la vision de la société (conservatrice ou oppositionnelle).

La représentation sociale constitue, certes, une notion moins statique que celle d'attitude ou de valeur, dans la mesure où elle dirige le chercheur vers les réseaux de significations variées que peuvent prendre divers objets sociaux ou situations selon les groupes ou classes sociales. L'étude des représentations sociales tente ainsi de réintroduire face aux prétentions à l'universalité des lois psychosociologiques, la diversité des objets, des conditions et des situations ${ }^{13}$. Elle se distingue notamment quant à l'importance qu'elle accorde à la pensée sociale par rapport à la pensée individuelle, c'est-à-dire aux modes de connaissance propres à différents groupes selon l'objet de représentation, les conditions de vie et les situations sociales particulières. On peut ainsi dépasser la connaissance du système de croyances et de valeurs individuelles, en introduisant les interactions multiples qui s'établissent entre l'individu et le contexte socioculturel dans lequel il se trouve inséré, véhiculant certaines idées, images et valeurs.

12. Moscovici, 1963 , p. 11.

13. Herzlich, 1972. 
Une telle approche dans la mesure où elle se situe au niveau sociétal, permet de mettre en évidence les relations sociales ou rapports de classes (et fractions de classe) qui se définissent autour d'un enjeu, c'est-à-dire d'une certaine image de la société et du changement. Touraine (1974) indique que dans nos sociétés occidentales actuelles les conflits sociaux et les luttes ne sont plus limités au domaine de la «production et de la propriété , mais se cristallisent autour du contrôle des moyens d'information et de consommation afin de "gérer » les transformations sociales et le changement, «c'est l'action sur l'environnement, c'est l'emprise du pouvoir orientant le changement sur l'ensemble de la vie sociale qui devient le lieu central des rapports et des conflits ${ }^{14}{ }^{\text {}}$. Notons ici rapidement que certaines perspectives exclusivement classistes ${ }^{15}$, réduisant l'acteur social à sa position de classe et à sa fonction dans la division du travail, ne rendent compte que d'une partie des conflits et des luttes. En effet si l'on accepte qu'actuellement, selon Touraine, la domination sociale et idéologique s'est étendue et "vise à modifier l'ensemble du comportement en fonction des objectifs et des formes de changement social ${ }^{16} \gg$, les forces d'opposition ne vont plus se limiter à la classe ouvrière mais à « un ensemble de minorités luttant contre l'emprise de la domination dans les secteurs les plus divers de la société. Cette hétérogénéité est d'autant plus grande que les forces d'opposition s'appuient, face au changement dirigé, sur des attributs sociaux et culturels non liés directement au rôle de production : âge, sexe, race, religion, etc. ${ }^{17} 》$. Il s'agirait donc de pouvoir établir les clivages socio-idéologiques, qui se dessinent dans la société canadienne, par exemple, selon les divers types de représentations et plus largement selon les images de la société (conservatrice ou oppositionnelle), pour identifier, non seulement les zones conflictuelles, mais également les forces agissantes ${ }^{18}$.

L'étude des représentations permet ainsi de dépasser l'approche traditionnelle des enquêtes d'attitudes trop exclusivement centrée sur l'analyse de l'organisation psychologique individuelle, négligeant les contraintes sociales et culturelles. En même temps

14. Touraine, 1974 , p. 187.

15. Voir Robert et Faugeron, 1977.

16. Touraine, 1976, p. 187.

17. Touraine, 1974, p. 190. de classes.

18. Il ne s'agit pas de nier ici le caractère prédominant des rapports 
elle fournit le chaînon manquant de l'enquête contextuelle — qui établit les liens objectifs entre les conditions de vie et les caractéristiques individuelles - en mettant en évidence les médiations symboliques et idéologiques spécifiques à une classe ou à un groupe et, au-delà, à une société par rapport auxquelles se déterminent les actions individuelles et collectives.

Mais là comme ailleurs le rapport individu-société est analysé comme un simple rapport de dépendance unilatéral, l'individu existant dans et par la totalité sociale. $\mathrm{La}$ réalité de l'individu et la réalité du groupe se construisent ensemble. Il ne s'agit pas de nier qu'il y ait dépendance, mais en posant d'emblée une telle dépendance, on ne la remet pas en question en la constituant comme problème de recherche. On peut reprendre ce que Crozier et Friedberg (1977) dans un autre domaine soulignent à propos d'une telle démarche surdéterministe : «... l'objet d'étude est réifié au lieu d'être constitué à travers le recherche même, il s'ensuit que [...] l'étude du devrait être prime la recherche sur ce qui est et sur la signification de ce qui est ${ }^{19}$ ». Il va alors s'agir, dans cette perspective, de s'interroger sur l'interaction de l'individu avec son environnement socioculturel, non pas comme une dépendance que l'on postule mais comme un problème à explorer et à comprendre. C'est à ce type de problème que s'adresse la sociologie phénoménologique, en particulier l'ethnométhodologie.

\subsection{La relation problématique individu-société}

Nous avons indiqué comment en partant de l'opinion qui constitue une réponse manifeste verbalisée, observable et donc mesurable mais en même temps labile, on peut arriver à une notion plus complexe : l'attitude, représentant une organisation latente relativement stable. Cette notion est utilisée pour rendre compte des liens entre stimuli et réponses, on la considère comme une réponse anticipée et une préparation à l'action. L'enquête contextuelle offre l'opportunité d'étudier simultanément les attitudes individuelles et les caractéristiques spécifiques du milieu de vie propre à chaque répondant. Elle permet ainsi de substituer, à des facteurs généraux arbitrairement sélectionnés, les particularités structurelles et les contraintes objectives réelles, liées aux situations et aux conditions de vie des répondants et qui circonscrivent

19. Crozier et Friedberg, p. 113, note infrapaginale. 
leur champ d'action. Enfin l'étude des représentations sociales introduit les médiations socioculturelles, comme construits idéologiques de la réalité faits par les différents groupes et classes sociales, et qui guident en partie leurs actions et réactions. Ces construits refiètent en effet les problèmes et difficultés propres à chacun de ces groupes ou classes sociales dans leurs rapports de pouvoir et de dépendance. Si l'on s'arrête là, on peut étudier de façon systématique les relations existantes entre différents facteurs contextuels, socioculturels et les structures d'attitudes pour établir des liens de causalité expliquant les variations d'attitudes empiriquement observables. Une telle analyse est certainement intéressante dans la mesure où elle permet de mettre en évidence la multiplicité des influences du contexte et de la culture sur les attitudes et de dépasser une approche centrée exclusivement sur les contraintes psychologiques et/ou les contraintes sociologiques abstraites (classe sociale, âge, etc.). Cependant elle reste limitée et sa valeur explicative faible dans la mesure où elle considère toujours l'individu comme déterminé par des contraintes intérieures et/ou extérieures. $\mathrm{Si}$ ces contraintes limitent la liberté d'action des acteurs, elles ne l'éliminent pas. On peut dire qu'elles constituent un cadre structurel en délimitant la marge d'autonomie.

Les approches conventionnelles évacuent trop facilement le problème d'autonomie en analysant de façon statique et déterministe les comportements. On prend comme évident le système social et la relation individu-société n'est jamais étudiée concrètement. Bien sûr de nombreuses hypothèses sont faites sur les processus à travers lesquels la structure de l'interaction sociale produit une réalité sociale émergente et sur le rôle que joue le système social sur les conduites. Mais les explications restent spéculatives, reposant sur un modèle à priori de la réalité, dans la mesure où il n'y a pas de données empiriques. Les variations qui sont mises en évidence décrivent mais n'expliquent pas concrètement ces différences, il manque une analyse réelle des processus à travers lesquels un système social émerge, est reconnu et intégré avec son mode de régulation.

\subsection{Sociologie phénoménologique et ethnométhodologie}

La sociologie phénoménologique et son récent développement l'ethnométhodologie offrent actuellement une démarche de recher- 
che extrêmement utile pour étudier de façon plus concrète et objective le problème de l'interdépendance individu-société et le mode de régulation de l'ordre social. Sans entrer ici dans les détails d'une approche qui se laisse difficilement définir en quelques lignes ${ }^{20}$, on peut indiquer la démarche générale. S'inspirant des travaux, notamment des philosophes allemands Husserl et Schutz et prolongeant les idées et la perspective de l'Interactionisme symbolique, l'ethnométhodologie ${ }^{21}$ va chercher à découvrir les règles implicites et explicites qu'utilisent les membres d'un groupe pour régler leurs interactions et maintenir un certain ordre social.

L'ethnométhodologie se différencie des autres approches traditionnelles, y compris l'Interactionisme symbolique dans la mesure où elle ne prend pas comme des données de fait les représentations, les valeurs, normes etc., mais pose leur existence même comme problème d'étude : comment, par quels moyens (ou procédures interprétatives et routines) l'homme de la rue comme le sociologue donnent sens, décrivent et s'assurent que des règles et des définitions existent? Les individus ne réalisent pas passivement les valeurs reçues et intériorisées mais utilisent activement certaines significations, certains instruments idéologiques et matériels disponibles, pour s'adapter à diverses situations, résoudre des problèmes qui surgissent. Elle dirige ainsi l'attention sur les règles ou procédures à travers lesquelles les membres d'un groupe social arrivent par exemple à définir et à interpréter une certaine conduite comme déviante. Comment ils décident «ce qui est arrivé », c'est-à-dire donnent sens, interprètent des objets ou des événements qu'ils rencontrent et choisissent d'agir d'une façon plutôt qu'une autre en fonction de la situation.

L'accent récent, sur le contrôle social et la réaction sociale par les sociologues de la déviance ${ }^{22}$, a mis en évidence les processus d'étiquetage et de stigmatisation. Mais, comme l'indique Phillipson (1972) : « The authors' use of each of these and others concepts in this area rests on their assumption that we know unequivocally to which processes they are referring [...] what is required is the careful detailed description of what moral - labels

20. Un article est en cours de rédaction par l'auteur sur l'utilisation de l'ethnométhodologie comme complément de l'analyse traditionnelle.

21. Voir notamment Garfinkel, 1967, et Cicourel, 1973, ainsi que Zimmerman et Pollner, 1970; Sacks, 1972 ; Wieder, 1973.

22. Voir par exemple Becker, 1963 ; Lemert, 1951 , etc. 
- means to those labelled, to the labellers, to the social audience and to sociologists ${ }^{23}$,

Si l'on considère la société comme un champ de créations conflictuelles ${ }^{24}$ ", où coexistent des idées, des valeurs et pratiques différentes sinon contradictoires, la relation sociétéindividu se pose avec une certaine acuité. On ne peut plus parler de détermination, mais bien au contraire d'indétermination et le problème alors est de comprendre pourquoi certains choix sont faits en fonction des capacités effectives des individus, des potentialités et opportunités que leur offre la situation. C'est en cela que l'ethnométhodologie, en proposant un nouveau paradigme, ouvre un champ d'étude dont l'exploration est nécessaire si l'on veut connaître les mécanismes réels d'adaptation et de changement et parvenir ensuite à expliquer concrètement l'organisation sociale et les modes de régulation et de contrôle.

\subsection{Vers une double approche macro et microsociologique}

Si l'on considère que la complexité des comportements humains ne se laisse pas facilement enfermer dans un modèle simple et déterministe, l'individu dans un système non totalitaire disposant toujours d'une certaine autonomie et liberté de choix selon les ressources qu'il a à sa disposition et ses capacités à s'en saisir, il ne s'agit pas non plus de tomber dans le piège de l'analyse centrée exclusivement sur les individus, leurs intentions et motivations pour expliquer leurs actions. En effet, les justifications données des actions le sont après coup et constituent souvent des rationalisations. Mais également, il reste que le système présente certaines contraintes structurelles qui ne sont pas toujours immédiatement conscientes pour les individus et offre un environnement culturel institué par, en même temps qu'en dehors, des acteurs. Il s'agit donc de pouvoir saisir comment les membres d'un groupe élaborent leur construction de la réalité, utilisent des règles et certains moyens à leur disposition pour créer, maintenir et altérer un ordre social. Mais en même temps, il est nécessaire d'établir les conditions qui préexistent et limitent les choix et les actions, c'est-à-dire les contraintes et opportunités structurelles du milieu dans lequel se situent les répondants (analyse contextuelle), ainsi que les construits socioculturels qui prédominent dans

23. Phillipson, 1972, p. 147.

24. Touraine, 1973. 
les différents groupes et classes sociales constituant ce qu'on pourrait appeler le champ culturel d'une société (étude des représentations sociales et des idéologies). L'ethnométhodologie ne remplace donc pas les analyses conventionnelles, mais constitue un complément pour formuler nos explications sur des données empiriques plutôt que sur des spéculations ou des résultats partiels. On peut ainsi réaliser ce que Stoetzel (1963) considère comme un des objectifs de la psychologie sociale, à savoir de développer des « explications rapportées à la fois au système culturel environnant, à la situation concrète des sujets qui réagissent et à la personnalité même de leurs auteurs ${ }^{25}$.

25. Stoetzel, 1963, p. 28. 


\section{BIBLIOGRAPHIE}

BECKER, H.S. (1963) : Outsiders, New York, The Free Press.

BERNSTEIN, B. (1964) : Elaborated and restricted codes : their social origins and some consequences $\gg$, in J.J. Gumperz et D. Hywes (cds), American Anthropologist, 66, part 2.

BLUMER, H. (1954) : «What is wrong with social theory », American Sociological Review, vol. XIX.

BLUMER, H. (1956) : Sociological Analysis and Variable *, American Sociological Review, vol. XXII.

BOUDON, R. (1971) : La crise de la sociologie : questions d'épistémologie sociologique, Genève, Suisse, Librairie Droz.

BOURDIEU, P. (1965) : Un art moyen, Paris, Editions de Minuit.

BOURDIEU, P. (1973) : «L'opinion publique n'existe pas », Les Temps Modernes, no 318 (janvier), p. 1292-1309.

CICOUREL, A.V. (1964) : Method and Measurement in Sociology, New York, The Free Press.

CICOUREL, A.V. (1973) : Cognitive sociology, Penguin Education.

CROZIER, M. et FRIEDBERG, E. (1977) : L'acteur et le système, Paris, Editions du Seuil.

GARFINKEL, H. (1967) : Studies in ethnomethodology, Englewood Cliffs, N.J., Prentice Hall.

HERZLICH, C. (1972) : «La représentation sociale» in S. Moscovici (ed.), Introduction d la psychologie sociale, Paris, Librairie Larousse, p. 303-325.

HENNESY, B.C. (1975) : Public Opinion, Belmont, California, Wadsworth Publication Company.

KLINEBERG, O., LOUIS-GUÉRIN, C. et ZAVALLONI, M. (1978) : Students, Values and Politics, New York, Free Press.

LAPIERE, R.T. (1934) : «Attitude vs. actions », Social Forces, 13, p. 230-237.

LEMERT, E.M. (1951) : Social Pathology, New York, McGraw Hill Book Co., Inc.

LIPSET, S.M., TROW, M. et COLEMAN, J.S. (1956) : Union democracy, Glencoe, The Free Press.

MOSCOVICI, S. (1961) : La psychanalyse, son image et son public, Paris, P.U.F.

MOSCOVICI, S. (1963) : Attitudes and opinions », Annual Review of Psychology, 14, p. 231-260.

PHILLIPSON, M. (1972) : « Theory, Methodology and Conceptualization », in P. Filner et al. (eds.), New Directions in Sociological Theory, Cambridge (Mass.), The M.I.T. Press, chap. 5.

ROBERT, Ph. et FAUGERON, C. (1977) : Note sur la recherche : représentations de la déviance, Paris, ronéo.

SACKS, H. (1972) : An Initial Investigation of the Usability of Conversational Data for Doing Sociology $\gg$, in D. Sundow (ed.), Studies in Interaction, New York, The Free Press. 
SCHATZMAN, L. et STRAUSS, A. (1955) : "Social Class and Modes of Communication >, American Journal of Sociology, Chicago (Iltinois), University of Chicago Press, vol. LX, no 4, p. 329-338.

STOETZEL, J. (1963) : La psychologie sociale, Paris, Flammarion.

TOURAINE, A. (1972) : Université et sociêté aux Etats-Unis, Paris, Éditions du Seuil.

TOURAINE, A. (1973) : Production de la société, Paris, Éditions du Seuil. TOURAINE, A. (1974) : Pour la sociologie, Paris, Éditions du Seuil.

WIEDER, D.L. (1973) : Language and Social Reality, La Haye, Mouton. ZIMMERMAN, D.H. et POLLNER, M. (1970) : « The Everyday world as phenomenon 》, in T. Douglas (ed.), Understanding Everyday Life, Chicago, Aldine. 